\title{
ON THE SCHRÖDINGER EQUATION \\ WITH STEPLIKE POTENTIALS
}

\author{
Tuncay Aktosun \\ Department of Mathematics \\ North Dakota State University \\ Fargo, ND 58105
}

\begin{abstract}
The one-dimensional Schrödinger equation is considered when the potential is asymptotic to a positive constant on the right half line in a certain sense. The zeroenergy limits of the scattering coefficients are obtained under weaker assumptions than used elsewhere, and the continuity of the scattering coefficients from the left are established. The scattering coefficients for the potential are expressed in terms of the corresponding coefficients for the pieces of the potential on the positive and negative half lines. The number of bound states for the whole potential is related to the number of bound states for the two pieces. Finally, an improved result is given on the small-energy asymptotics of reflection coefficients for potentials supported on a half line.
\end{abstract}

PACS Numbers: 03.65.Nk, 03.80.+r

Mathematics Subject Classification (1991): 34L25, 34L40, 81U05

Keywords: 1-D Schrödinger equation, Nondecaying potentials, Steplike potentials, Zeroenergy limit, Continuity of scattering coefficients, Asymptotics of scattering coefficients Short title: Schrödinger equation with steplike potentials 


\section{INTRODUCTION}

Consider the one-dimensional Schrödinger equation

$$
\psi^{\prime \prime}(k, x)+k^{2} \psi(k, x)=V(x) \psi(k, x), \quad x \in \mathbf{R},
$$

where the potential $V$ is real valued and may be written as $V=V_{1}+V_{2}$ such that $V_{1}$ has support in $\mathbf{R}^{-}, V_{2}$ has support in $\mathbf{R}^{+}$, and

$$
V_{1} \in L_{1}^{1}\left(\mathbf{R}^{-}\right), \quad V_{2}-c^{2} \in L_{1}^{1}\left(\mathbf{R}^{+}\right),
$$

for some positive $c$, where the prime denotes the derivative with respect to the spatial variable $x, \mathbf{R}^{-}=(-\infty, 0), \mathbf{R}^{+}=(0,+\infty)$, and $L_{\alpha}^{1}(I)$ is the set of measurable functions $f$ on an interval $I$ such that $\int_{I} d x(1+|x|)^{\alpha}|f(x)|$ is finite. We will use $\mathbf{C}^{+}$to denote the upper half complex plane and $\overline{\mathbf{C}^{+}}=\mathbf{C}^{+} \cup \mathbf{R}$.

The scattering problem for (1.1) consists of the analysis of the scattering coefficients corresponding to the potential $V$. Such an analysis was given by Buslaev and Fomin ${ }^{1}$ and by Cohen and Kappeler; ${ }^{2}$ however, in Ref. 1 only the generic case was considered, and in Ref. 2 the exceptional case was considered under the stronger assumption of $L_{2}^{1}$ instead of $L_{1}^{1}$ in (1.2). The definition of an exceptional potential is given in the next section; informally speaking, an exceptional potential has a 'zero-energy bound state,' or equivalently it is at the boundary of changing the number of its bound states by one. The bound states of (1.1) are its square-integrable solutions.

Our primary aim is to consider the small $k$-asymptotics of the scattering coefficients in the exceptional case and prove their continuity at $k=0$ by assuming only (1.2). One consequence of our analysis is that under (1.2), the number of bound states is finite. We present a Levinson theorem relating the number of bound states to the zero-energy limit of the phase of the transmission coefficient, and relate the scattering coefficients corresponding to $V, V_{1}$ and $V_{2}$ to each other. 
The inverse scattering problem for (1.1) is equivalent to the recovery of the potential in terms of an appropriate set of scattering data. Such problems were analyzed in Refs. 1-3. Our result in Theorem 3.4 is expected to have an impact on the inverse scattering theory for (1.1) because it was used as a hypothesis in Ref. 2 to obtain various results in the analysis of the inverse scattering problem for (1.1).

Our results are also expected to have an impact on the phase recovery problem, a version of the inverse scattering problem for (1.1) with inportant applications ${ }^{4-6}$ in the recovery of material properties of thin films. Mathematically speaking, one is interested in the recovery of $V_{2}$ by using only $V_{1}$ and the reflectivity measurements, i.e. the amplitudes of reflection coefficients without their phases. In reality, the phase of the complex-valued reflection coefficient cannot be measured, even though the reflectivity is easily measured ${ }^{5-8}$ by using a device known as a reflectometer. Our analysis of the scattering coefficients for $V$ in terms of those for $V_{1}$ and $V_{2}$ helps us to solve the phase recovery problem by the so-called two-layer method $\mathrm{d}^{9,10}$ using $33 \%$ less data than the so-called three-layer method. ${ }^{9,11-13}$

Our paper is organized as follows. In Section II we introduce the Jost solutions and scattering coefficients for (1.1), explain the distinction between the generic and exceptional cases, and obtain the small- $k$ asymptotics of the Jost solution from the left and of its $x$ derivative. In Section III, in the exceptional case, we prove that the Wronskian defined in (2.10) vanishes linearly as $k \rightarrow 0$ in $\overline{\mathbf{C}^{+}}$; the proof is nontrivial, but the result is significant and it enables us to obtain the small- $k$ asymptotics of the scattering coefficients and establish the continuity at $k=0$ of the scattering coefficients from the left. In Section IV we present a Levinson theorem, relating the number of bound states to the zero-energy phase of the transmission coefficient. Section V explores the relation among the scattering coefficients for $V, V_{1}$, and $V_{2}$. In Section VI the small- $k$ limits of the scattering coefficients for $V_{1}$ and $V_{2}$ are given, and in Section VII such limits are related to the corresponding limits for $V$. In Section VII it is also shown that, except for one special case, one can 
derive the small- $k$ limits of the scattering coefficients for $V$ in terms of the corresponding limits for $V_{1}$ and $V_{2}$; in the special case, namely when both $V_{1}$ and $V_{2}$ are generic and $V$ is exceptional, for such a derivation one needs to know that (3.52) holds for some nonzero $\alpha$ even though the value of $\alpha$ is not needed. In Section VII we also relate the number of bound states for $V$ to the corresponding numbers for $V_{1}$ and $V_{2}$, and show that the former number is one less than or equal to the sum of the numbers of bound states for $V_{1}$ and $V_{2}$. Finally, in Section VIII, when $c=0$ in (1.2), we present an improved result on the small- $k$ asymptotics of the reflection coefficient from the right (left) for a potential supported on the left (right) half line in the generic case; this is done by reconsidering the special case in Section VI and relating the value of $\alpha$ in (3.52) to the parameters corresponding to $V_{1}$ and $V_{2}$. The small-energy expansions of the reflection coefficients given in Section VIII are expected to simplify various proofs in the direct and inverse scattering theory for the Schrödinger equation with potentials belonging to $L_{1}^{1}(\mathbf{R})$.

\section{PRELIMINARIES}

The scattering states of (1.1) correspond to solutions behaving like $e^{ \pm i k x}$ as $x \rightarrow-\infty$ and like $e^{ \pm i \gamma x}$ as $x \rightarrow+\infty$, where

$$
\gamma=\sqrt{k^{2}-c^{2}}
$$

and the branch of the square root function is used with $\operatorname{Im} \gamma \geq 0$. Thus, when $k \in(-c, c)$, $\gamma$ defined in (2.1) is purely imaginary and is given by $\gamma=i \sqrt{c^{2}-k^{2}}$. The mapping $k \mapsto \gamma$ is analytic from $\mathbf{C}^{+}$to itself and is continuous on $\overline{\mathbf{C}^{+}}$. The inverse mapping $\gamma \mapsto k$ is analytic only in $\gamma \in \mathbf{C}^{+} \backslash i(0, c]$ and is continuous only in $\gamma \in \overline{\mathbf{C}^{+}} \backslash i[0, c)$.

The Jost solution from the left, $f_{l}(k, x)$, associated with $V$ is the solution of (1.1) satisfying

$$
e^{-i \gamma x} f_{l}(k, x)=1+o(1), \quad e^{-i \gamma x} f_{l}^{\prime}(k, x)=i \gamma+o(1), \quad x \rightarrow+\infty .
$$


Similarly, $f_{r}(k, x)$, the Jost solution from the right, is defined as the solution of (1.1) satisfying

$$
e^{i k x} f_{r}(k, x)=1+o(1), \quad e^{i k x} f_{r}^{\prime}(k, x)=-i k+o(1), \quad x \rightarrow-\infty
$$

The transmission and reflection coefficients from the left, $T$ and $L$, can be defined in terms of the spatial asymptotics of $f_{l}(k, x)$ as

$$
e^{-i k x} f_{l}(k, x)=\frac{1}{T(k)}+\frac{L(k)}{T(k)} e^{-2 i k x}+o(1), \quad x \rightarrow-\infty
$$

Similarly, the transmission and reflection coefficients from the right can be defined by using the asymptotics of $f_{r}(k, x)$ as $x \rightarrow+\infty$; however, these coefficients can be expressed ${ }^{1,2}$ in terms of $T$ and $L$, and they are not essential in our analysis. We will never need the transmission coefficient from the right which is equal to $\gamma T(k) / k$, and the reflection coefficient from the right is used only in Theorem 3.5 and is defined in (3.53). If $c=0$ then transmission coefficients from the left and from the right are the same, but they are different if $c \neq 0$. Further properties of these coefficients can be found in Refs. 1 and 2 .

In terms of the Jost solutions, we define the Faddeev functions $m_{l}(k, x)$ and $m_{r}(k, x)$ :

$$
m_{l}(k, x)=e^{-i \gamma x} f_{l}(k, x), \quad m_{r}(k, x)=e^{i k x} f_{r}(k, x) .
$$

From (2.2), (2.3), and (2.4) it follows that

$$
\begin{gathered}
m_{l}(k, x)=1+\frac{1}{2 i \gamma} \int_{x}^{\infty} d y\left[e^{2 i \gamma(y-x)}-1\right]\left[V(y)-c^{2}\right] m_{l}(k, y) \\
m_{l}^{\prime}(k, x)=-\int_{x}^{\infty} d y e^{2 i \gamma(y-x)}\left[V(y)-c^{2}\right] m_{l}(k, y) \\
m_{r}(k, x)=1+\frac{1}{2 i k} \int_{-\infty}^{x} d y\left[e^{2 i k(x-y)}-1\right] V(y) m_{r}(k, y)
\end{gathered}
$$




$$
m_{r}^{\prime}(k, x)=\int_{-\infty}^{x} d y e^{2 i k(x-y)} V(y) m_{r}(k, y) .
$$

Proposition 2.1 Assume (1.2) is satisfied for some $c>0$. Then, for each fixed $x \in \mathbf{R}$, the functions $f_{l}(k, x)$ and $f_{l}^{\prime}(k, x)$ are analytic in $\gamma \in \mathbf{C}^{+}$. Consequently, as $k \rightarrow 0$ in $\overline{\mathbf{C}^{+}}$, we have

$$
f_{l}(k, 0)=f_{l}(0,0)+O\left(k^{2}\right), \quad f_{l}^{\prime}(k, 0)=f_{l}^{\prime}(0,0)+O\left(k^{2}\right) .
$$

PROOF: The analyticity in $\gamma \in \mathbf{C}^{+}$can be proved by iterating the Volterra integrals (2.5) and (2.6) and using (2.4). By (2.1), $k=0$ corresponds to $\gamma=i c$. Expanding $f_{l}(k, 0)$ and $f_{l}^{\prime}(k, 0)$ in $\gamma$ at $\gamma=i c$, we obtain $(2.9)$.

Define

$$
W(k):=\frac{2 i k}{T(k)}=\left[f_{r}(k, x) ; f_{l}(k, x)\right],
$$

where $[f ; g]=f g^{\prime}-f^{\prime} g$ denotes the Wronskian. Recall that the Wronskian of any two solutions of (1.1) is independent of $x$ and depends only on $k$. Generically, $W(0) \neq 0$, and $f_{l}(0, x)$ and $f_{r}(0, x)$ are linearly independent. In the exceptional case, $f_{l}(0, x)$ and $f_{r}(0, x)$ are linearly dependent and hence $W(0)=0$. We will say that $V$ is a generic (exceptional) potential if the generic (exceptional) case occurs. By $(2.4),(2.5)$, and $(2.7)$, both $f_{l}(0, x)$ and $f_{r}(0, x)$ are real valued. In the exceptional case, there exists a real nonzero constant $\alpha$ such that

$$
\alpha=\frac{f_{l}(0, x)}{f_{r}(0, x)} .
$$

\section{ANALYSIS OF $W(k)$ IN THE EXCEPTIONAL CASE}

In this section we analyze $W(k)$ in the exceptional case and show that $W(k) / k$ has a nonzero limit as $k \rightarrow 0$. The existence of such a limit was used as a hypothesis in many 
theorems in Ref. 2, and it was proved there only under the stronger assumption $L_{2}^{1}$ instead of $L_{1}^{1}$ used in (1.2). Our aim is to evaluate this limit under (1.2) alone. For the proof we proceed as in the Appendix of Ref. 14, where the method was first used in Ref. 15 for the Schrödinger equation with $c=0$.

As a first step, let us define the solutions of $(1.1), s(k, x)$ and $v(k, x)$, satisfying the boundary conditions

$$
s(k, 0)=1, \quad s^{\prime}(k, 0)=0 ; \quad v(k, 0)=0, \quad v^{\prime}(k, 0)=1 .
$$

In fact, these solutions satisfy

$$
\begin{gathered}
s(k, x)=\left\{\begin{array}{l}
\cos k x+\frac{1}{k} \int_{x}^{0} d y \sin k(y-x) V(y) s(k, y), \quad x \leq 0, \\
\cos \gamma x+\frac{1}{\gamma} \int_{0}^{x} d y \sin \gamma(x-y)\left[V(y)-c^{2}\right] s(k, y), \quad x \geq 0,
\end{array}\right. \\
v(k, x)=\left\{\begin{array}{l}
\frac{\sin k x}{k}+\frac{1}{k} \int_{x}^{0} d y \sin k(y-x) V(y) v(k, y), \quad x \leq 0, \\
\frac{\sin \gamma x}{\gamma}+\frac{1}{\gamma} \int_{0}^{x} d y \sin \gamma(x-y)\left[V(y)-c^{2}\right] v(k, y), \quad x \geq 0 .
\end{array}\right.
\end{gathered}
$$

Note also that

$$
\begin{aligned}
& s^{\prime}(k, x)=\left\{\begin{array}{l}
-k \sin k x-\int_{x}^{0} d y \cos k(y-x) V(y) s(k, y), \quad x \leq 0, \\
-\gamma \sin \gamma x+\int_{0}^{x} d y \cos \gamma(x-y)\left[V(y)-c^{2}\right] s(k, y), \quad x \geq 0,
\end{array}\right. \\
& v^{\prime}(k, x)=\left\{\begin{array}{l}
\cos k x-\int_{x}^{0} d y \cos k(y-x) V(y) v(k, y), \quad x \leq 0, \\
\cos \gamma x+\int_{0}^{x} d y \cos \gamma(x-y)\left[V(y)-c^{2}\right] v(k, y), \quad x \geq 0 .
\end{array}\right.
\end{aligned}
$$

Using (3.1) we get

$$
f_{l}(k, 0)=\left[f_{l}(k, x) ; v(k, x)\right], \quad f_{l}^{\prime}(k, 0)=-\left[f_{l}(k, x) ; s(k, x)\right],
$$




$$
f_{r}(k, 0)=\left[f_{r}(k, x) ; v(k, x)\right], \quad f_{r}^{\prime}(k, 0)=-\left[f_{r}(k, x) ; s(k, x)\right] .
$$

From (3.2) and (3.4), as $x \rightarrow \pm \infty$ we obtain

$$
\begin{aligned}
& s(k, x)=\frac{e^{i k x} A_{1}(k)}{2 i k}+\frac{e^{-i k x} A_{2}(k)}{2 i k}+o(1), \quad x \rightarrow-\infty, \\
& s^{\prime}(k, x)=\frac{e^{i k x} A_{1}(k)}{2}-\frac{e^{-i k x} A_{2}(k)}{2}+o(1), \quad x \rightarrow-\infty,
\end{aligned}
$$

$$
s(k, x)=\frac{e^{i \gamma x} A_{3}(k)}{2 i \gamma}+\frac{e^{-i \gamma x} A_{4}(k)}{2 i \gamma}+o(1), \quad x \rightarrow+\infty,
$$

$$
s^{\prime}(k, x)=\frac{e^{i \gamma x} A_{3}(k)}{2}-\frac{e^{-i \gamma x} A_{4}(k)}{2}+o(1), \quad x \rightarrow+\infty,
$$

where we have defined

$$
\begin{gathered}
A_{1}(k)=i k-\int_{-\infty}^{0} d y e^{-i k y} V(y) s(k, y), \\
A_{2}(k)=i k+\int_{-\infty}^{0} d y e^{i k y} V(y) s(k, y), \\
A_{3}(k)=i \gamma+\int_{0}^{\infty} d y e^{-i \gamma y}\left[V(y)-c^{2}\right] s(k, y), \\
A_{4}(k)=i \gamma-\int_{0}^{\infty} d y e^{i \gamma y}\left[V(y)-c^{2}\right] s(k, y) .
\end{gathered}
$$

Similarly, from (3.3) and (3.5), as $x \rightarrow \pm \infty$ we obtain

$$
\begin{gathered}
v(k, x)=\frac{e^{i k x} A_{5}(k)}{2 i k}-\frac{e^{-i k x} A_{6}(k)}{2 i k}+o(1), \quad x \rightarrow-\infty, \\
v^{\prime}(k, x)=\frac{e^{i k x} A_{5}(k)}{2}+\frac{e^{-i k x} A_{6}(k)}{2}+o(1), \quad x \rightarrow-\infty,
\end{gathered}
$$




$$
v(k, x)=\frac{e^{i \gamma x} A_{7}(k)}{2 i \gamma}-\frac{e^{-i \gamma x} A_{8}(k)}{2 i \gamma}+o(1), \quad x \rightarrow+\infty,
$$

$$
v^{\prime}(k, x)=\frac{e^{i \gamma x} A_{7}(k)}{2}+\frac{e^{-i \gamma x} A_{8}(k)}{2}+o(1), \quad x \rightarrow+\infty,
$$

where we have defined

$$
\begin{gathered}
A_{5}(k)=1-\int_{-\infty}^{0} d y e^{-i k y} V(y) v(k, y), \\
A_{6}(k)=1-\int_{-\infty}^{0} d y e^{i k y} V(y) v(k, y), \\
A_{7}(k)=1+\int_{0}^{\infty} d y e^{-i \gamma y}\left[V(y)-c^{2}\right] v(k, y), \\
A_{8}(k)=1+\int_{0}^{\infty} d y e^{i \gamma y}\left[V(y)-c^{2}\right] v(k, y) .
\end{gathered}
$$

Evaluating the Wronskians in (3.6) as $x \rightarrow+\infty$ and by using (2.2), (3.10), (3.11), (3.16), and (3.17), we get

$$
f_{l}(k, 0)=A_{8}(k), \quad f_{l}^{\prime}(k, 0)=A_{4}(k) .
$$

Similarly, evaluating the Wronskians in (3.7) as $x \rightarrow-\infty$ and by using (2.3), (3.8), (3.9), (3.14), and (3.15), we have

$$
f_{r}(k, 0)=A_{5}(k), \quad f_{r}^{\prime}(k, 0)=-A_{1}(k) .
$$

Now let $\phi(k, x)$ be the solution of (1.1) satisfying

$$
\phi(k, 0)=f_{l}(0,0), \quad \phi^{\prime}(k, 0)=f_{l}^{\prime}(0,0) .
$$

For the arguments in the rest of this section, there is no loss of generality in assuming that $f_{l}(0,0) \neq 0$; if $f_{l}(0,0)=0$, the proofs can be modified as in Ref. 14 to get the results given 
in Theorems 3.4 and 3.5. Because $\phi(0, x)$ and $f_{l}(0, x)$ are solutions of the same differential equation with the same initial conditions given in (3.22), we have

$$
\phi(0, x)=f_{l}(0, x), \quad x \in \mathbf{R} \text {. }
$$

Using (2.11) and (3.23) we see that in the exceptional case $\phi(0, x)$ remains bounded as $x \rightarrow \pm \infty$; this is because $f_{l}(0, x)$ and $f_{r}(0, x)$ remain bounded as $x \rightarrow+\infty$ and $x \rightarrow-\infty$, respectively. From (3.1) and (3.22) it follows that

$$
\phi(k, x)=f_{l}(0,0) s(k, x)+f_{l}^{\prime}(0,0) v(k, x) .
$$

Our aim is to express $W(k)$ defined in (2.10) in terms of $\phi(k, x)$. Evaluating the Wronskian in (2.10) at $x=0$ and using (3.20) and (3.21), we obtain

$$
f_{l}(0,0) W(k)=f_{r}(k, 0) f_{l}(0,0) A_{4}(k)+f_{l}(k, 0) f_{l}(0,0) A_{1}(k) .
$$

Using (3.12), (3.18), (3.21), and (3.24), we have

$$
f_{l}(0,0) A_{1}(k)=i k f_{l}(0,0)+f_{l}^{\prime}(0,0)-f_{l}^{\prime}(0,0) f_{r}(k, 0)-\int_{-\infty}^{0} d y e^{-i k y} V(y) \phi(k, y)
$$

Similarly, using (3.13), (3.19), (3.20), and (3.24), we get

$$
f_{l}(0,0) A_{4}(k)=i \gamma f_{l}(0,0)-f_{l}^{\prime}(0,0)+f_{l}^{\prime}(0,0) f_{l}(k, 0)-\int_{0}^{\infty} d y e^{i \gamma y}\left[V(y)-c^{2}\right] \phi(k, y) .
$$

Thus, using (3.26) and (3.27) in (3.25), we obtain

$$
f_{l}(0,0) W(k)=-f_{r}(k, 0) M_{1}(k)+f_{l}(k, 0) M_{2}(k),
$$

where

$$
M_{1}(k)=-i \gamma f_{l}(0,0)+f_{l}^{\prime}(0,0)+\int_{0}^{\infty} d y e^{i \gamma y}\left[V(y)-c^{2}\right] \phi(k, y)
$$




$$
M_{2}(k)=i k f_{l}(0,0)+f_{l}^{\prime}(0,0)-\int_{-\infty}^{0} d y e^{-i k y} V(y) \phi(k, y) .
$$

Proposition 3.1 Assume (1.2) is satisfied for some $c>0$. Then, $M_{1}(k)$ defined in (3.29) is an analytic function of $\gamma \in \mathbf{C}^{+}, M_{1}(0)=0$, and

$$
M_{1}(k)=O\left(k^{2}\right), \quad k \rightarrow 0 \text { in } \overline{\mathbf{C}^{+}} .
$$

PROOF: Using (3.20), (3.27)-(3.29), we see that $M_{1}(k)$ is a linear combination of $f_{l}(k, 0)$ and $f_{l}^{\prime}(k, 0)$, and in fact

$$
M_{1}(k)=-f_{l}(0,0) f_{l}^{\prime}(k, 0)+f_{l}^{\prime}(0,0) f_{l}(k, 0) .
$$

Thus, by Proposition 2.1 and (3.32), $M_{1}(k)$ is analytic in $\gamma \in \mathbf{C}^{+}$. Using its Taylor series expansion around $\gamma=i c$, which corresponds to $k=0$, we obtain (3.31) and see that $M_{1}(0)=0$.

In the following proposition and elsewhere, we will use $C$ to denote a generic positive constant whose value is not necessarily the same in different appearances.

Proposition 3.2 Assume that we are in the exceptional case and that (1.2) holds for some $c \geq 0$. Then,

$$
|\phi(k, x)-\phi(0, x)| \leq C\left(\frac{|k x|}{1+|k x|}\right)^{2}, \quad x \leq 0,
$$

with $k \in[-\epsilon, \epsilon]$ for any fixed positive $\epsilon$.

PROOF: Using (3.1), (3.22), and (3.24) we obtain

$\phi(k, x)=f_{l}(0,0) \cos k x+f_{l}^{\prime}(0,0) \frac{\sin k x}{k}+\frac{1}{k} \int_{x}^{0} d y \sin k(y-x) V(y) \phi(k, y), \quad x \leq 0$,

$$
\phi(0, x)=f_{l}(0,0)+x f_{l}^{\prime}(0,0)+\int_{x}^{0} d y(y-x) V(y) \phi(0, y), \quad x \leq 0 .
$$


Since $\phi(0, x)$ remains bounded as $x \rightarrow-\infty$, the linear growth in $x$ in (3.35) as $x \rightarrow-\infty$ cannot happen and we must have

$$
f_{l}^{\prime}(0,0)-\int_{-\infty}^{0} d y V(y) \phi(0, y)=0
$$

and thus

$$
\phi(0, x)=f_{l}(0,0)+\int_{-\infty}^{0} d y y V(y) \phi(0, y)+o(1), \quad x \rightarrow-\infty .
$$

From (3.34)-(3.36), we get

$$
\phi(k, x)-\phi(0, x)=I_{1}+I_{2}+I_{3}+I_{4}+I_{5}+\frac{1}{k} \int_{x}^{0} d y \sin k(y-x) V(y)[\phi(k, y)-\phi(0, y)]
$$

where we have defined

$$
\begin{gathered}
I_{1}=x\left[\frac{\sin k x}{k x}-1\right] \int_{-\infty}^{x} d y V(y) \phi(0, y), \\
I_{2}=[\cos k x-1] f_{l}(0,0), \quad I_{3}=\frac{\sin k x}{k} \int_{x}^{0} d y[1-\cos k y] V(y) \phi(0, y), \\
I_{4}=-(1-\cos k x) \int_{x}^{0} d y \frac{\sin k y}{k} V(y) \phi(0, y), \\
I_{5}=\int_{x}^{0} d y y\left[\frac{\sin k y}{k y}-1\right] V(y) \phi(0, y) .
\end{gathered}
$$

For $z \geq 0$, the function $z \mapsto z /(1+z)$ is monotone increasing and we have

$$
|\sin z| \leq \frac{C z}{1+z}, \quad\left|1-\frac{\sin z}{z}\right| \leq \frac{C z^{2}}{(1+z)^{2}}, \quad|1-\cos z| \leq \frac{C z^{2}}{(1+z)^{2}} .
$$

Hence, for $x \leq 0$ and $k \in[-\epsilon, \epsilon]$, from (3.39)-(3.42) we get the estimates

$$
\left|I_{j}\right| \leq \frac{C|k x|^{2}}{(1+|k x|)^{2}}, \quad j=1,2,3,4,5
$$


Using (3.43) and (3.44) in (3.38), we obtain

$$
|\phi(k, x)-\phi(0, x)| \leq \frac{C|k x|^{2}}{(1+|k x|)^{2}}+\frac{C|k x|}{1+|k x|} \int_{x}^{0} d y|V(y)||\phi(k, y)-\phi(0, y)|,
$$

With the help of Gronwall's lemma, from (3.45) we get (3.33).

Proposition 3.3 Assume $V$ is an exceptional potential and (1.2) holds for some $c>0$, and let $M_{2}(k)$ be the quantity defined in (3.30). Then, as $k \rightarrow 0$ on the real axis, we have

$$
f_{l}(k, 0) M_{2}(k)=i k \alpha f_{l}(0,0)+o(k),
$$

where $\alpha$ is the real nonzero constant defined in (2.11).

PROOF: Using (3.30) and (3.36) we get

$$
f_{l}(k, 0) M_{2}(k)=f_{l}(k, 0)\left[i k f_{l}(0,0)+J_{1}+J_{2}\right]
$$

where

$$
\begin{gathered}
J_{1}=-\int_{-\infty}^{0} d y\left[e^{-i k y}-1\right] V(y) \phi(0, y), \\
J_{2}=-\int_{-\infty}^{0} d y e^{-i k y} V(y)[\phi(k, y)-\phi(0, y)] .
\end{gathered}
$$

Because of $(3.23)$, in the exceptional case $\phi(0, y)$ is bounded for $y \leq 0$. Using the inequality

$$
\left|e^{i z}-i z-1\right| \leq \frac{C z^{2}}{1+z}, \quad z \geq 0
$$

from (3.48) we get

$$
J_{1}=i k \int_{-\infty}^{0} d y y V(y) \phi(0, y)+o(k)
$$

Moreover, using (3.33) in (3.49) we get

$$
\left|J_{2}\right| \leq C|k| \int_{-\infty}^{0} d y \frac{|k y|}{1+|k y|}(-y)|V(y)|
$$


which gives us $J_{2}=o(k)$. Hence, using (2.9) and (3.50) in (3.47), we obtain

$$
f_{l}(k, 0) M_{2}(k)=i k f_{l}(0,0)^{2}+i k f_{l}(0,0) \int_{-\infty}^{0} d y y V(y) \phi(0, y)+o(k) .
$$

Using (3.37) we can explicitly evaluate the integral on the right hand side of (3.51). Since $f_{r}(0, x)=1+o(1)$ as $x \rightarrow-\infty$, with the help of $(2.11),(3.23)$, and (3.37), we get

$$
\int_{-\infty}^{0} d y y V(y) \phi(0, y)=\alpha-f_{l}(0,0)
$$

and hence $(3.51)$ reduces to $(3.46)$.

Theorem 3.4 Assume that $V$ is an exceptional potential and that (1.2) holds for some $c>0$. Then, $W(0)=0$ and

$$
W(k)=i \alpha k+o(k), \quad k \rightarrow 0 \text { in } \overline{\mathbf{C}^{+}},
$$

where $\alpha$ is the real nonzero constant given by (2.11).

PROOF: Using (3.28), (3.31), and (3.46), we see that (3.52) holds as $k \rightarrow 0$ through real values. However, using the Phragmén-Lindelöf theorems as on p. 2997 of Ref. 14, it follows that the limit is valid also when $k \rightarrow 0$ in $\overline{\mathbf{C}^{+}}$.

The reflection coefficient from the right for (1.1), $R$, is related to $T$ and $L$ as ${ }^{1,2}$

$$
R(k)=-\frac{L(k)^{*} T(k)}{T(k)^{*}}, \quad k \in \mathbf{R} \backslash\{0\},
$$

where the asterisk denotes complex conjugation. The continuity of $T, L$, and $R$ at $k=0$ is already known ${ }^{2}$ in the generic case under (1.2). Next, we show that their continuity holds also in the exceptional case.

Theorem 3.5 Assume that $V$ is an exceptional potential and that (1.2) holds for some $c>0$. Then, the scattering coefficients $T, L$, and $R$ are all continuous at $k=0$, and we have

$$
T(k)=\frac{2}{\alpha}+o(1), \quad k \rightarrow 0 \text { in } \overline{\mathbf{C}^{+}}
$$




$$
L(k)=1+o(1), \quad R(k)=-1+o(1), \quad k \rightarrow 0 \text { in } \mathbf{R},
$$

where $\alpha$ is the real nonzero constant given in (2.11).

PROOF: From (2.10) and Theorem 3.4, we get (3.54), which also proves the continuity of $T$ at $k=0$. Using (3.53) and the identity ${ }^{1,2}$

$$
L(k)=\frac{T(k)}{T(k)^{*}}, \quad k \in[-c, c] \backslash\{0\},
$$

and the fact that $\alpha$ is real and nonzero, we get (3.55) and the continuity of $L$ and $R$ at $k=0$.

\section{THE LEVINSON THEOREM}

In Theorem 3.5 we have proved the continuity of $T$ and $L$ at $k=0$ in the exceptional case. In the generic case, the continuity of these functions is already known ${ }^{2}$ and also follows from (2.10) and (3.56), which lead to

$$
\begin{gathered}
T(k)=\frac{2 i k}{W(0)}+o(k), \quad k \rightarrow 0 \text { in } \overline{\mathbf{C}^{+}}, \\
L(k)=-1+o(1), \quad k \rightarrow 0 \text { in } \mathbf{R} .
\end{gathered}
$$

The continuity of $T$ at $k=0$ indicates that the poles of $T$ cannot accumulate at $k=0$, which assures the finiteness of such poles. Proceeding as in Theorem 9.1 of Ref. 16 we obtain the following analog of the Levinson theorem.

Theorem 4.1 Assume that $V$ satisfies (1.2) for some $c \geq 0$. Then the number of bound states of (1.1) is finite and given by

$$
N=\frac{d}{2}+\frac{1}{\pi}\left[\arg T\left(0^{+}\right)\right],
$$


where $d=0$ in the exceptional case and $d=1$ in the generic case, and $\arg T(k)$ denotes the continuous branch of the argument of $T$ normalized such that $\arg T(+\infty)=0$.

PROOF: The continuity of $T$ at $k=0$ assures us that $k=0$ cannot be an accumulation point for the poles of $T$ in $\mathbf{C}^{+}$. It is already $\mathrm{known}^{1,2}$ that such poles are simple and confined to the positive imaginary axis, $1 / T$ is continuous in $\overline{\mathbf{C}^{+}} \backslash\{0\}$, and $T(k)=1+O(1 / k)$ as

$k \rightarrow \infty$ in $\overline{\mathbf{C}^{+}}$. Thus, we have all the ingredients to proceed as in the proof of Theorem 9.1 of Ref. 16.

Proposition 4.2 Assume that $V$ satisfies (1.2) for some $c>0$. Then, the real nonzero constant $\alpha$ defined in (2.11) in the exceptional case has the same sign as that of $e^{i N \pi}$. The sign of $W(0)$ in the generic case is the same as the sign of $e^{i(N+1) \pi}$.

PROOF: In the exceptional case, comparing (3.54) and (4.3) gives us the sign of $\alpha$. In the generic case, comparing (4.1) and (4.3) we get the sign of $W(0)$.

\section{FACTORIZATION}

Let $V_{j}$ denote $V_{1}$ and $V_{2}$ for $j=1$ and $j=2$, respectively. We will use $T_{j}, L_{j}$, and $R_{j}$ for the transmission coefficient from the left, the reflection coefficient from the left, and the reflection coefficient from the right, respectively, for the potential $V_{j}$. Similarly, let $f_{l ; j}(k, x)$ and $f_{r ; j}(k, x)$ denote the Jost solutions from the left and from the right, respectively, for $V_{j}$. As in (2.10) we will use $W_{j}(k)$ to denote $2 i k / T_{j}(k)$; in the exceptional case, as in (2.11) we will use $\alpha_{j}$ to denote the nonzero real constant $f_{l ; j}(0, x) / f_{r ; j}(0, x)$. We will also let $N_{j}$ denote the number of bound states of $V_{j}$.

Proposition 5.1 Assume that $V$ satisfies (1.2) for some $c \geq 0$. Then,

$$
\frac{1}{T(k)}=\frac{1-R_{1}(k) L_{2}(k)}{T_{1}(k) T_{2}(k)}, \quad k \in \mathbf{R} \backslash\{0\}
$$




$$
\frac{L(k)}{T(k)}=\frac{L_{2}(k)-R_{1}(k)^{*}}{T_{1}(k)^{*} T_{2}(k)}, \quad k \in \mathbf{R} \backslash\{0\}
$$

The result stated in Proposition 5.1 holds when only $L^{1}$ is used instead of $L_{1}^{1}$ in (1.2); however, we will take the limit in (5.1) and (5.2) as $k \rightarrow 0$ and hence it is more convenient to have the result stated under (1.2). Proposition 5.1 is a special case of the following factorization result whose proof can be given as in Refs. 17 and 18. Let us partition the real axis $\mathbf{R}$ into $p$ fragments as $\mathbf{R}=\cup_{j=1}^{p}\left(x_{j-1}, x_{j}\right)$, where $x_{0}=-\infty, x_{p}=+\infty$, and $x_{j-1}<x_{j}$ for $j=1, \cdots, p$. We can then write the potential $V$ in terms of its fragments $V_{j-1, j}$ as

$$
V(x)=\sum_{j=1}^{p} V_{j-1, j}(x)
$$

where we have defined

$$
V_{j-1, j}(x)= \begin{cases}V(x), & x \in\left(x_{j-1}, x_{j}\right), \\ 0, & x \notin\left(x_{j-1}, x_{j}\right) .\end{cases}
$$

Note that $V_{j-1, j} \in L^{1}(\mathbf{R})$ for $j=1, \cdots, p-1$, and the right-most fragment $V_{p-1, p}$ satisfies $V_{p-1, p}-c^{2} \in L^{1}(\mathbf{R})$. Let $T_{j-1, j}$ and $L_{j-1, j}$ denote the transmission and reflection coefficients from the left, respectively, for $V_{j-1, j}$. Let us define the transition matrix $\Lambda$ associated with $V$ and $\Lambda_{j-1, j}$ associated with $V_{j-1, j}$ as

$$
\Lambda(k)=\left[\begin{array}{cc}
\frac{1}{T(k)} & \frac{L(k)^{*}}{T(k)^{*}} \\
\frac{L(k)}{T(k)} & \frac{1}{T(k)^{*}}
\end{array}\right], \quad \Lambda_{j-1, j}(k)=\left[\begin{array}{cc}
\frac{1}{T_{j-1, j}(k)} & \frac{L_{j-1, j}(k)^{*}}{T_{j-1, j}(k)^{*}} \\
\frac{L_{j-1, j}(k)}{T_{j-1, j}(k)} & \frac{1}{T_{j-1, j}(k)^{*}}
\end{array}\right] .
$$

From (3.56) and the identity ${ }^{2}$

$$
1-|L(k)|^{2}=\frac{\gamma}{k}|T(k)|^{2}, \quad k \in \mathbf{R} \backslash(-c, c),
$$

it follows that the determinant of $\Lambda$ is given by

$$
\operatorname{det} \Lambda(k)= \begin{cases}\frac{\gamma}{k}, & k \in \mathbf{R} \backslash(-c, c), \\ 0, & 0<|k| \leq c .\end{cases}
$$


The two columns in each of $\Lambda$ and $\Lambda_{p, p+1}$ are identical when $0<|k| \leq c$.

Theorem 5.2 Assume $V$ satisfies (1.2) for some $c \geq 0$, where $L^{1}$ is used instead of $L_{1}^{1}$. Let $\Lambda$ be the transition matrix corresponding to the potential $V$ and let $\Lambda_{j-1, j}$ correspond to the fragment $V_{j-1, j}$ defined in (5.4). Then, we have

$$
\Lambda(k)=\Lambda_{0,1}(k) \Lambda_{1,2}(k) \cdots \Lambda_{p-1, p}(k), \quad k \in \mathbf{R} \backslash\{0\} .
$$

The result in Proposition 5.1 corresponds to $p=2$ in Theorem 5.2 by using the $(1,1)$ and $(2,1)$ entries in the matrix equality in $(5.5)$ and that $R_{1}(k)=-L_{1}(k)^{*} T_{1}(k) / T_{1}(k)^{*}$ for $k \in \mathbf{R} \backslash\{0\}$.

\section{ASYMPTOTICS OF SCATTERING COEFFICIENTS FOR $V_{1}$ AND $V_{2}$}

In considering the potential $V_{1}$, the analog of Theorem 4.1 states that

$$
\arg T_{1}\left(0^{+}\right)=\left(N_{1}-\frac{d_{1}}{2}\right) \pi
$$

where $d_{1}=0$ if $V_{1}$ is exceptional and $d_{1}=1$ if $V_{1}$ is generic. Using the boundary conditions at $x=0$ based on the continuity of $f_{r ; 1}(k, x)$ and $f_{r ; 1}^{\prime}(k, x)$, we have

$$
\frac{1+R_{1}(k)}{T_{1}(k)}=f_{r ; 1}(k, 0), \quad i k \frac{-1+R_{1}(k)}{T_{1}(k)}=f_{r ; 1}^{\prime}(k, 0),
$$

where $f_{r ; 1}(k, x)$ is the Jost solution from the right for $V_{1}$. Thus, from (6.2) it follows that

$$
W_{1}(k):=\frac{2 i k}{T_{1}(k)}=i k f_{r ; 1}(k, 0)-f_{r ; 1}^{\prime}(k, 0) .
$$

Using the general theory ${ }^{19-21}$, or with the help of $(2.4),(2.7)$, and (2.8) we have

$$
f_{r ; 1}(k, 0)=f_{r ; 1}(0,0)+o(1), \quad f_{r ; 1}^{\prime}(k, 0)=f_{r ; 1}^{\prime}(0,0)+o(1), \quad k \rightarrow 0 \text { in } \overline{\mathbf{C}^{+}}
$$

and hence $(6.3)$ and $(6.4)$ give us $W_{1}(0)=-f_{r ; 1}^{\prime}(0,0)$. Generically $W_{1}(0) \neq 0$, and in the exceptional case we have $W_{1}(0)=0$. Thus, generically we obtain

$$
W_{1}(k)=-f_{r ; 1}^{\prime}(0,0)+o(1), \quad k \rightarrow 0 \text { in } \overline{\mathbf{C}^{+}}
$$


and hence from (6.2) and (6.3) we get

$$
R_{1}(k)=-1+T_{1}(k) f_{r ; 1}(k, 0)=-1-2 i k \mu_{1}+o(k), \quad k \rightarrow 0 \text { in } \overline{\mathbf{C}^{+}},
$$

where we have defined

$$
\mu_{1}=\frac{f_{r ; 1}(0,0)}{f_{r ; 1}^{\prime}(0,0)}
$$

Note that $\mu_{1}$ is well defined because $f_{r ; 1}^{\prime}(0,0)=-W_{1}(0) \neq 0$ when $V_{1}$ is generic. In Section VIII we will improve the result in (6.6) by evaluating the next term in the expansion. Comparing (6.1) and (6.5) we see that the sign of $f_{r ; 1}^{\prime}(0,0)$ is the same as the sign of $e^{i N_{1} \pi}$. Moreover, with the help of (2.3) we get

$$
f_{r ; 1}(k, x)=f_{r}(k, x), \quad f_{r ; 1}^{\prime}(k, x)=f_{r}^{\prime}(k, x), \quad x \leq 0 .
$$

Now let us turn to the exceptional case. In this case, under $V_{1} \in L_{1}^{1}\left(\mathbf{R}^{-}\right)$, it is known that $W_{1}(k)$ vanishes linearly in $k$ as $k \rightarrow 0$ in $\overline{\mathbf{C}^{+}}$. We have ${ }^{22}$

$$
\begin{gathered}
W_{1}(k)=\frac{i k\left(\alpha_{1}^{2}+1\right)}{\alpha_{1}}+o(k), \quad k \rightarrow 0 \text { in } \overline{\mathbf{C}^{+}} \\
T_{1}(k)=\frac{2 \alpha_{1}}{\alpha_{1}^{2}+1}+o(1), \quad k \rightarrow 0 \text { in } \overline{\mathbf{C}^{+}} \\
R_{1}(k)=-\frac{\alpha_{1}^{2}-1}{\alpha_{1}^{2}+1}+o(1), \quad k \rightarrow 0 \text { in } \overline{\mathbf{C}^{+}}
\end{gathered}
$$

where $\alpha_{1}$ is the real nonzero constant given by

$$
\alpha_{1}=\frac{f_{l ; 1}(0, x)}{f_{r ; 1}(0, x)} .
$$


Comparing (6.1) and (6.9) we see that the sign of $\alpha_{1}$ is the same as the sign of $e^{i N_{1} \pi}$. Note that $\frac{\alpha_{1}^{2}-1}{\alpha_{1}^{2}+1}$ is an increasing function of $\alpha_{1}^{2}$ and its values are confined to the interval $(-1,1)$. Thus, $R_{1}(0) \in(-1,1)$ in the exceptional case.

Let us now summarize some similar results for $V_{2}$, where (1.2) holds for some $c>0$. From Theorem 4.1 we have

$$
\arg T_{2}\left(0^{+}\right)=\left(N_{2}-\frac{d_{2}}{2}\right) \pi
$$

where $d_{2}=0$ for the exceptional case and $d_{2}=1$ in the generic case. Using the continuity of the Jost solution $f_{l ; 2}(k, x)$ and its derivative $f_{l ; 2}^{\prime}(k, x)$ at $x=0$, we get

$$
\frac{1+L_{2}(k)}{T_{2}(k)}=f_{l ; 2}(k, 0), \quad i k \frac{1-L_{2}(k)}{T_{2}(k)}=f_{l ; 2}^{\prime}(k, 0) .
$$

As in Proposition 2.1 we have

$$
f_{l ; 2}(k, 0)=f_{l ; 2}(0,0)+O\left(k^{2}\right), \quad f_{l ; 2}^{\prime}(k, 0)=f_{l ; 2}^{\prime}(0,0)+O\left(k^{2}\right), \quad k \rightarrow 0 \text { in } \overline{\mathbf{C}^{+}}
$$

where $f_{l ; 2}(0,0)$ and $f_{l ; 2}^{\prime}(0,0)$ cannot simultaneously vanish because of $(2.2)$. Moreover, from (2.2) we obtain

$$
f_{l ; 2}(k, x)=f_{l}(k, x), \quad f_{l ; 2}^{\prime}(k, x)=f_{l}^{\prime}(k, x), \quad x \geq 0 .
$$

With the help of (6.12), defining

$$
W_{2}(k):=\frac{2 i k}{T_{2}(k)}=i k f_{l ; 2}(k, 0)+f_{l ; 2}^{\prime}(k, 0)
$$

from (6.13) we have

$$
W_{2}(k)=f_{l ; 2}^{\prime}(0,0)+i k f_{l ; 2}(0,0)+O\left(k^{2}\right), \quad k \rightarrow 0 \text { in } \overline{\mathbf{C}^{+}} .
$$

The generic case occurs if $W_{2}(0) \neq 0$; therefore, generically we have $f_{l ; 2}^{\prime}(0,0) \neq 0$, and in the exceptional case we have $f_{l ; 2}^{\prime}(0,0)=0$. Thus, generically, from $(6.15)$ and $(6.16)$ we get

$$
T_{2}(k)=\frac{2 i k}{f_{l ; 2}^{\prime}(0,0)}+\frac{2 \mu_{2} k^{2}}{f_{l ; 2}^{\prime}(0,0)}+O\left(k^{3}\right), \quad k \rightarrow 0 \text { in } \overline{\mathbf{C}^{+}}
$$


where $\mu_{2}$ is the real constant defined as

$$
\mu_{2}=\frac{f_{l ; 2}(0,0)}{f_{l ; 2}^{\prime}(0,0)}
$$

Comparing (6.11) and (6.17), we conclude that the sign of $f_{l ; 2}^{\prime}(0,0)$ is the same as the sign of $e^{i\left(N_{2}+1\right) \pi}$. With the help of (6.12) and (6.17) we also get

$$
L_{2}(k)=-1+2 i k \mu_{2}+2 k^{2} \mu_{2}^{2}+O\left(k^{3}\right), \quad k \rightarrow 0 \text { in } \overline{\mathbf{C}^{+}}
$$

In the exceptional case we have $W_{2}(0)=0$, i.e. $f_{l ; 2}^{\prime}(0,0)=0$ and $f_{l ; 2}(0,0) \neq 0$. In this case it follows from (6.16) that $W_{2}(k)$ vanishes linearly in $k$ as $k \rightarrow 0$ in $\overline{\mathbf{C}^{+}}$. From (6.15) we get

$$
\begin{gathered}
W_{2}(k)=i k f_{l ; 2}(0,0)+O\left(k^{2}\right), \quad k \rightarrow 0 \text { in } \overline{\mathbf{C}^{+}}, \\
T_{2}(k)=\frac{2}{f_{l ; 2}(0,0)}+O(k)=\frac{2}{\alpha_{2}}+O(k), \quad k \rightarrow 0 \text { in } \overline{\mathbf{C}^{+}},
\end{gathered}
$$

where $\alpha_{2}$ is the real nonzero constant given by

$$
\alpha_{2}=\frac{f_{l ; 2}(0, x)}{f_{r ; 2}(0, x)}
$$

and we have used the fact that $f_{r ; 2}(0, x)=1$ for $x \leq 0$. Comparing (6.11) and (6.20), we see that the sign of $\alpha_{2}$ is the same as the sign of $e^{i N_{2} \pi}$. Using (6.12) and (6.20), we obtain

$$
L_{2}(k)=1+O(k), \quad k \rightarrow 0 \text { in } \overline{\mathbf{C}^{+}}
$$

\section{ASYMPTOTICS OF SCATTERING COEFFICIENTS FOR $V$}

In this section, using the results in Section VI, with the help of (5.1) and (5.2), we will derive the small- $k$ asymptotics of $T$ and $L$ and compare our results with those obtained in (4.1) (4.2), and Theorem 3.5. 
With the help of (5.1), let

$$
F(k):=\frac{T_{1}(k) T_{2}(k)}{T(k)}=1-R_{1}(k) L_{2}(k),
$$

and let $\omega(k)$ denote the phase of $F(k)$ as normalized in Theorem 4.1. From (7.1) we get

$$
\omega\left(0^{+}\right)=\arg T_{1}\left(0^{+}\right)+\arg T_{2}\left(0^{+}\right)-\arg T\left(0^{+}\right)
$$

Using (4.3), (6.1), and (6.11) in (7.2), we obtain

$$
\omega\left(0^{+}\right)=\left(N_{1}+N_{2}-N-\frac{d_{1}+d_{2}-d}{2}\right) \pi
$$

If both $V_{1}$ and $V_{2}$ are exceptional, from (6.10), (6.21), and (7.1) we get

$$
F(k)=\frac{2 \alpha_{1}^{2}}{\alpha_{1}^{2}+1}+o(1), \quad k \rightarrow 0 \text { in } \overline{\mathbf{C}^{+}}
$$

and hence $\omega\left(0^{+}\right)=0$. Using $(6.9),(6.20),(7.1)$, and (7.4) we have

$$
T(k)=\frac{2}{\alpha_{1} \alpha_{2}}+o(1), \quad k \rightarrow 0 \text { in } \overline{\mathbf{C}^{+}}
$$

Thus, $V$ is exceptional, $N=N_{1}+N_{2}, T\left(0^{+}\right)$is real and nonzero, and the sign of $T\left(0^{+}\right)$ is the same as that of $e^{i\left(N_{1}+N_{2}\right) \pi}$, where the latter fact is obtained by using $(7.5)$ and the signs of $\alpha_{1}$ and $\alpha_{2}$ determined in Section VI. Using (5.2), (6.9), (6.10), (6.20), (6.21), and (7.5) we also get

$$
L(k)=1+o(1), \quad k \rightarrow 0 \text { in } \mathbf{R} .
$$

If $V_{1}$ is exceptional and $V_{2}$ is generic, then using $(6.9),(6.10),(6.17),(6.19)$, and (7.1), we obtain

$$
\begin{gathered}
F(k)=\frac{2}{\alpha_{1}^{2}+1}+o(1), \quad k \rightarrow 0 \text { in } \overline{\mathbf{C}^{+}} \\
T(k)=\frac{2 i \alpha_{1} k}{f_{l ; 2}^{\prime}(0,0)}+o(k), \quad k \rightarrow 0 \text { in } \overline{\mathbf{C}^{+}}
\end{gathered}
$$


In this case, $V$ is generic and $\omega\left(0^{+}\right)=0$, and hence from (7.3) we get $N=N_{1}+N_{2}$. With the help of (5.2) we also obtain

$$
L(k)=-1+o(1), \quad k \rightarrow 0 \text { in } \mathbf{R} .
$$

If $V_{1}$ is generic and $V_{2}$ is exceptional, then using (6.5), (6.6), (6.20), (6.21), and (7.1) we get

$$
\begin{gathered}
F(k)=2+O(k), \quad k \rightarrow 0 \text { in } \overline{\mathbf{C}^{+}}, \\
T(k)=-\frac{2 i k}{\alpha_{2} f_{r ; 1}^{\prime}(0,0)}+o(k), \quad k \rightarrow 0 \text { in } \overline{\mathbf{C}^{+}}
\end{gathered}
$$

and hence $V$ is generic, $\omega\left(0^{+}\right)=0$, and from (7.3) it follows that $N=N_{1}+N_{2}$. With the help of (5.2) we also obtain

$$
L(k)=-1+o(1), \quad k \rightarrow 0 \text { in } \mathbf{R} .
$$

If both $V_{1}$ and $V_{2}$ are generic, using (6.5)-(6.8), (6.14), (6.17)-(6.19), and (7.1) we get

$$
F(k)=2 i k\left(\mu_{2}-\mu_{1}\right)+o(k)=-\frac{2 i k W(0)}{f_{r ; 1}^{\prime}(0,0) f_{l ; 2}^{\prime}(0,0)}+o(k), \quad k \rightarrow 0 \text { in } \overline{\mathbf{C}^{+}}
$$

where $W(k)$ is the Wronskian given in $(2.10)$ because

$$
W(k)=\left[f_{r}(k, x) ; f_{l}(k, x)\right]=f_{r ; 1}(k, 0) f_{l ; 2}^{\prime}(k, 0)-f_{r ; 1}^{\prime}(k, 0) f_{l ; 2}(k, 0) .
$$

Thus, we have two possibilities, namely $W(0) \neq 0$ and $W(0)=0$. If $W(0) \neq 0$, then $V$ is generic, and in this case using (5.1), (5.2), (6.5), (6.6), (6.17), (6.19), (7.1), and (7.6) we get

$$
\begin{gathered}
T(k)=\frac{2 i k}{W(0)}+o(k), \quad k \rightarrow 0 \text { in } \overline{\mathbf{C}^{+}}, \\
L(k)=-1+o(1), \quad k \rightarrow 0 \text { in } \mathbf{R},
\end{gathered}
$$


agreeing with (4.1) and (4.2). From (4.3) and (7.8) it is seen that $W(0)$ has the same sign as the sign of $e^{i(N+1) \pi}$. From (7.6), since $W(0) \neq 0$, we get $\omega\left(0^{+}\right)= \pm \pi / 2$. In Section VI we have seen that the sign of $f_{r ; 1}^{\prime}(0,0)$ is the same as that of $e^{i N_{1} \pi}$ and the sign of $f_{l ; 2}^{\prime}(0,0)$ is the same as that of $e^{i\left(N_{2}+1\right) \pi}$. Thus, in the subcase $\mu_{2}>\mu_{1}$ with $\omega\left(0^{+}\right)=\pi / 2$, from (7.3) we get $N=N_{1}+N_{2}-1$. Similarly, in the subcase $\mu_{2}<\mu_{1}$ with $\omega\left(0^{+}\right)=-\pi / 2$, we obtain $N=N_{1}+N_{2}$.

If both $V_{1}$ and $V_{2}$ are generic and $W(0)=0$, then $V$ is exceptional. In this case, without consulting Theorem 3.4, by using (6.5), (6.6), (6.17), and (6.19) we can only conclude that $F(k)=o(k)$ as $k \rightarrow 0$. If we knew the expansion in (6.6) up to $o\left(k^{2}\right)$, then we would have determined $F(k)$ up to $o\left(k^{2}\right)$ as well. If we use Theorem 3.4, with the help of (2.11), (3.52), and (7.1) we get

$$
\begin{gathered}
F(k)=\frac{2 \alpha k^{2}}{f_{r ; 1}^{\prime}(0,0) f_{l ; 2}^{\prime}(0,0)}+o\left(k^{2}\right), \quad T(k)=\frac{2}{\alpha}+o(1), \quad k \rightarrow 0 \text { in } \overline{\mathbf{C}^{+}}, \\
L(k)=1+o(1), \quad k \rightarrow 0 \text { in } \mathbf{R},
\end{gathered}
$$

where, by (4.3), the sign of $\alpha$ is the same as that of $e^{i N \pi}$. Since $\left|R_{1}(k)\right|<1$ for $k \in \mathbf{R} \backslash\{0\}$, we must have $\omega\left(0^{+}\right)=0$. In this case, with the help of (7.3), we get $N=N_{1}+N_{2}-1$.

The above analysis shows that $N=N_{1}+N_{2}$ or $N=N_{1}+N_{2}-1$. As in Theorem 2.1 of Ref. 22, using induction we obtain the following general result.

Theorem 7.1 Assume $V$ satisfying (1.2) for some $c \geq 0$ is partitioned into $p$ fragments as in (5.3), and let $N_{j-1, j}$ denote the number of bound states corresponding to $V_{j-1, j}$. Then

$$
1-p+\sum_{j=1}^{p} N_{j-1, j} \leq N \leq \sum_{j=1}^{p} N_{j-1, j}, \quad p=1,2, \ldots
$$

\section{SMALL-ENERGY ASYMPTOTICS OF $R_{1}(k)$}

In this section, we will improve the asymptotics in (6.6). We will obtain the small- $k$ asymptotics of the reflection coefficients for potentials supported on a half line up to $o\left(k^{2}\right)$. 
The results given here are expected to contribute to better understanding of the scattering and inverse scattering theory for the Schrödinger equation with $c=0$, and this is explored in Ref. 23 .

Theorem 8.1 Assume $V_{1}$ is supported in $\mathbf{R}^{-}$and $V_{1} \in L_{1}^{1}\left(\mathbf{R}^{-}\right)$. Then, in the generic case we have

$$
R_{1}(k)=-1-2 i k \mu_{1}+2 k^{2}\left[\mu_{1}^{2}+\frac{1}{f_{r ; 1}^{\prime}(0,0)^{2}}\right]+o\left(k^{2}\right), \quad k \rightarrow 0 \text { in } \overline{\mathbf{C}^{+}},
$$

where $\mu_{1}$ is the quantity defined in (6.7).

PROOF: Given the generic potential $V_{1}$, let us choose $V_{2}$ satisfying (1.2) with $c>0$ such that $\mu_{2}=\mu_{1}$, where $\mu_{2}$ is the quantity defined in (6.18). As seen from (7.6), this corresponds to having $V_{2}$ generic and $V$ exceptional. In this case, using (7.7) at $k=0$, with the help of (2.11), (6.8), and (6.14), we obtain $\alpha=f_{l ; 2}^{\prime}(0,0) / f_{r ; 1}^{\prime}(0,0)$. Thus, (7.9) gives us

$$
F(k)=\frac{2 k^{2}}{f_{r ; 1}^{\prime}(0,0)^{2}}+o\left(k^{2}\right), \quad k \rightarrow 0 \text { in } \overline{\mathbf{C}^{+}} .
$$

On the other hand, using $\mu_{2}=\mu_{1}$ in (6.19) we get

$$
L_{2}(k)=-1+2 i k \mu_{1}+2 k^{2} \mu_{1}^{2}+O\left(k^{3}\right), \quad k \rightarrow 0 \text { in } \overline{\mathbf{C}^{+}} .
$$

Because of (7.1) we have

$$
R_{1}(k)=\frac{1-F(k)}{L_{2}(k)}
$$

and using (8.2) and (8.3) in (8.4) we get (8.1).

When $c=0$, the Taylor series expansion in (6.19) is no longer valid. However, we can use the analog of Theorem 8.1 and use the transformation $x \mapsto-x$ to obtain the following result. 
Corollary 8.2 Assume $V_{2}$ is supported in $\mathbf{R}^{+}$and $V_{2} \in L_{1}^{1}\left(\mathbf{R}^{+}\right)$, i.e. assume that $c=0$ in (1.2). Then, in the generic case we have

$$
L_{2}(k)=-1+2 i k \mu_{2}+2 k^{2}\left[\mu_{2}^{2}+\frac{1}{f_{l ; 2}^{\prime}(0,0)^{2}}\right]+o\left(k^{2}\right), \quad k \rightarrow 0 \text { in } \overline{\mathbf{C}^{+}},
$$

where $\mu_{2}$ is the quantity defined in (6.18).

Acknowledgments. The author is indebted to Martin Klaus for his comments. The research leading to this article was supported in part by the National Science Foundation under grant DMS-9803219.

\section{REFERENCES}

$1 \mathrm{~V}$ Buslaev and V. Fomin, An inverse scattering problem for the one-dimensional Schrödinger equation on the entire axis, Vestnik Leningrad. Univ. 17, 56-64 (1962) (Russian).

2 A. Cohen and T. Kappeler, Scattering and inverse scattering for steplike potentials in the Schrödinger equation, Indiana Univ. Math. J. 34, 127-180 (1985).

3 J. Legendre, Problème inverse de Schrödinger sur la ligne avec conditions dissymmetriques et applications, Ph.D. thesis, Acad. Montpellier, Univ. Sci. Tech. Languedoc, 1982.

4 X.-L. Zhou and S.-H. Chen, Theoretical foundations of X-ray and neutron reflectometry, Phys. Rep. 257, 223-348 (1995).

${ }^{5}$ G. Felcher and T. Russell (eds.), Proceedings of the workshop on methods of analysis and interpretation of neutron reflectivity data, Phys. B 173 (1991).

${ }^{6}$ G. Felcher and H. You (eds.), Proceedings of the 4th international conference on surface X-ray and neutron scattering, Phys. B 221 (1996). 
7 G. P. Felcher, Of butterflies and terraces, Neutron News 5, 18-22 (1994).

${ }^{8}$ V. O. de Haan, A. A. van Well, S. Adenwalla, and G. P. Felcher, Retrieval of phase information in neutron reflectometry, Phys. Rev. B 52, 10831-10833 (1995).

9 T. Aktosun and P. Sacks, Inversion of reflectivity data for nondecaying potentials, preprint (1999) [the manuscript is available from the author's home page at the url http://www.math.ndsu.nodak.edu/faculty/aktosun/preprints.html].

10 T. Aktosun and P. Sacks, Phase recovery with nondecaying potentials, in preparation (1999).

11 C. F. Majkrzak and N. F. Berk, Exact determination of the phase in neutron reflectometry, Phys. Rev. B 52, 10827-10830 (1995).

12 C. F. Majkrzak and N. F. Berk, Exact determination of the neutron reflection amplitude or phase, Phys. B 221, 520-523 (1996).

13 V. O. de Haan, A. A. van Well, P. E. Sacks, S. Adenwalla, and G. P. Felcher, Toward the solution of the inverse problem in neutron reflectometry, Phys. B 221, 524-532 (1996).

14 T. Aktosun, M. Klaus, and C. van der Mee. Inverse wave scattering with discontinuous wave speed, J. Math. Phys. 36, 2880-2928 (1995).

15 M. Klaus, Low-energy behaviour of the scattering matrix for the Schrödinger equation on the line, Inverse Probl. 4, 505-512 (1988).

${ }^{16}$ T. Aktosun, M. Klaus, and C. van der Mee, Wave scattering in one dimension with absorption, J. Math. Phys. 39, 1957-1992 (1998).

17 T. Aktosun, A factorization of the scattering matrix for the Schrödinger equation and 
for the wave equation in one dimension, J. Math. Phys. 33, 3865-3869 (1992).

18 T. Aktosun, M. Klaus, and C. van der Mee, Factorization of scattering matrices due to partitioning of potentials in one-dimensional Schrödinger-type equations, J. Math. Phys. 37, 5897-5915 (1996).

19 L. D. Faddeev, Properties of the S-matrix of the one-dimensional Schrödinger equation, Amer. Math. Soc. Transl. 2, 139-166 (1964) [Trudy Mat. Inst. Steklova 73, 314-336 (1964) (Russian)].

${ }^{20}$ P. Deift and E. Trubowitz, Inverse scattering on the line, Comm. Pure Appl. Math. 32, 121-251 (1979).

${ }^{21}$ K. Chadan and P. C. Sabatier, Inverse problems in quantum scattering theory, 2nd ed., Springer, New York, 1989.

22 T. Aktosun, M. Klaus, and C. van der Mee, On the number of bound states for the 1-D Schrödinger equation, J. Math. Phys. 39, 4249-4256 (1998).

${ }^{23}$ T. Aktosun, M. Klaus, and C. van der Mee, On the inverse scattering problem for the 1-D Schrödinger equation, in preparation (1999). 